\title{
10 Die männliche Genitalbeschneidung
}

\author{
Matthias Franz
}

\subsection{Geschichtlich-kultureller Hintergrund}

Die Beschneidung der männlichen Vorhaut ist wahrscheinlich die älteste und mit Sicherheit die am häufigsten durchgeführte operative Intervention überhaupt. Die Erhellung der vorgeschichtlichen Hintergründe der männlichen Genitalbeschneidung jenseits der Darstellung und Erwähnung in etwa 5.0oo Jahre alten altägyptischen Artefakten und deutlich jüngeren Texten der drei abrahamitischen Religionen ist Gegenstand humanethologischer, anthropologischer sowie religions- und kulturgeschichtlicher Forschung. Diese haben aufgrund schwieriger Beleglagen notwendigerweise auch spekulativen Charakter.

Das an Jungen oder jugendlichen Männern vollzogene Beschneidungsritual könnte in prähistorischen Jägerkulturen im Sinne eines männlichen Initiationsritus der Aggressionskontrolle oder zur demonstrativen Absicherung patriarchalisch geprägter Loyalitätsforderungen und Triebkontrolle sowie zur Abgrenzung der Stammesidentität gedient haben (vgl. Franz 2010). Möglicherweise wurde die Beschneidung als archaisches Rechtsinstitut genutzt, um die patriarchalische Aggressions- und Triebkontrolle innerhalb der Bezugsgruppe zu gewährleisten. Dies könnte unter den Bedingungen der Alltagspräsenz aggressiver Handlungszwänge eine adaptive und sinnvolle Sanktionsandrohung zur Eindämmung destruktiver Handlungsimpulse innerhalb der Bezugsgruppe gewesen sein. 
Es liegen Berichte von beschnittenen indianischen Ureinwohnern Südamerikas vor, auch in afrikanischen, vorderasiatisch-orientalischen Kulturen oder bei den australischen Ureinwohnern wurde und wird die männliche Genitalbeschneidung praktiziert, was die möglicherweise weltweite Verbreitung dieser Praxis bereits in prähistorischen Zeiten nahelegen könnte. Altägyptische Quellen belegen die Jünglingsbeschneidung. Möglicherweise fungierte die männliche Genitalbeschneidung auch im Sinne einer Körpertrophäe als Mindervariante der Tötung des Feindes oder der praktizierten Kastration nach kriegerischer Gefangennahme oder auch zur Kennzeichnung von Sklaven(vgl. Alanis u. Lucidi 2004). Hinweise auf ähnliche kriegerische Praktiken finden sich auch in Texten des Alten Testaments, z.B. in den Geschichten von der Bluttat von Sichem (Gen 34) oder der Tötung von 200 Philistern durch David, der deren abgetrennte Vorhäute dem König Saul als Brautpreis beibringen musste (1 Sam 18, 25-27).

Religionsgeschichtlich wird die am achten Lebenstag des neugeborenen Jungen stattfindende rituelle jüdische Genitalbeschneidung als ein zivilisatorisch progressives Äquivalent des ebenfalls am achten Tage zu erbringenden archaischen Opfers des erstgeborenen Sohnes diskutiert.

„Das aber ist mein Bund, den ihr halten sollt zwischen mir und euch und deinem Geschlecht nach dir: Alles, was männlich ist unter euch, soll beschnitten werden; eure Vorhaut sollt ihr beschneiden. Das soll das Zeichen sein des Bundes zwischen mir und euch. Jedes Knäblein, wenn's acht Tage alt ist, sollt ihr beschneiden bei euren Nachkommen." (Gen 17, 10-12)

Am achten Tag nach der Geburt wurde auch das archaische Erstlingsopfer dargebracht:

„Den Ertrag deines Feldes und den Überfluss deines Weinberges sollst du nicht zurückhalten. Deinen ersten Sohn sollst du mir geben. So sollst du auch tun mit deinem Stier und deinem Kleinvieh. Sieben Tage lass es bei seiner Mutter sein, am achten Tage sollst du es mir geben." (Ex22, 28-29)

Das Opfer der männlichen Erstgeburt, das auch noch in der Geschichte von der Tötung der ägyptischen erstgeborenen Kinder vor dem Auszug der Israeliten aus Ägypten anklingt (Ex 11, 4-6), wurde in Israel später nur noch an Tieren und schließlich nur im Zentralheiligtum des Jerusalemer Tempels von der Priesterschaft vollzogen.

Schon im spätbronzezeitlichen Kulturkreis des südöstlichen Mittelmeerraumes erstreckte sich die Praxis des dem Baal Kanaans oder in Karthago auch der Tanit dargebrachten phönizischen Kindesopfers (molk; im Alten Testament als Molochopfer erwähnt) bis hinein ins Karthago des zweiten vorchristlichen Jahrhunderts (Huss 1994). Im Weiteren erfolgte eine intensive, mythologisch konservierte Auseinandersetzung mit dem Kindesopfer und dessen Transformation in die stellvertretende Opferung von Tieren (z.B. das als molchomor in- 
schriftlich bezeugte punische Ersatzopfer eines Lammes). Die kulturelle Auseinandersetzung der zugewanderten israelitischen Anhänger des monotheistischen Jahweglaubens mit der in Kanaan bereits ansässigen westsemitischen und phönizischen Bevölkerung führte auf zahlreichen Gebieten zu einer Durchdringung und Entlehnung kanaanäischer Elemente (Kaiser 1970). So wurde die Opferung von Kindern für den kanaanitischen Baal zeitweise auch in Israel praktiziert. Von König Ahas in Juda (741-725 v. Chr.) wie auch von König Manasse (696-642 v. Chr.) wird berichtet, dass sie ihre Söhne „durchs Feuer gehen“ ließen (2 Kön 16, 3 bzw. 2 Kön 21, 6). Bereits die vom Erzvater Abraham zunächst in Angriff genommene Opferung des Erstgeborenen erscheint in der erst im letzten Moment von Gott abgelehnten Tötung Isaaks als kaum verstelltes, aber eigentlich noch intendiertes archaisches Kindesopfer. Das Brandopfer des Erstgeborenen wurde im späteren Judentum, insbesondere in nachexilischer Zeit, sanktioniert (Lev 20, 2-5; 2 Kön 23, 10) und durch das Tieropfer abgelöst.

Die drei abrahamitischen Religionen, das Judentum, das Christentum und der Islam, beziehen sich alle auf den Topos des heiligen Blutopfers, speziell auf das des erstgeborenen Sohnes, welches in verschiedenen Varianten und Opferpraktiken (Beschneidung, Tieropfer, Opferfest, Kreuzigung mit Auferstehung, Abendmahl) abgeschwächt oder modifiziert wurde, in der kulturellen Latenz aber weiter wirksam ist. Im Sinne eines ethnisch exklusiven Zeichens göttlicher Verbundenheit und Opferbereitschaft dient die obligatorische Beschneidung aller neugeborenen Jungen beispielsweise im Judentum als transgenerational tradierte, verletzende und bleibende Körpereinschreibung auch der verpflichtenden Festigung der gruppal-religiösen Identität:

„Wenn aber ein Männlicher nicht beschnitten wird an seiner Vorhaut, wird er ausgerottet werden aus seinem Volk, weil er meinen Bund gebrochen hat." (Gen 17, 14)

Nach Meinung des Autors macht der Subtext von Genesis 17, mit dem die Beschneidung eingeführt wird, die implizite patriarchalische Gewalt der Beschneidung sehr deutlich: Abraham erhält nach einigen Verfehlungen die Erlaubnis, zahlreiche Kinder - also generativen Sex - zu haben. Aber nur zu den Bedingungen des Gottes, der von Abraham als Bundeszeichen der Loyalität dessen Vorhaut fordert und damit eine offensichtliche Kastrationsandrohung etabliert. Gott wird in Genesis 17 ungewöhnlicherweise im Hebräischen als „El Shaddai“ bezeichnet. Religionsgeschichtlich und ethymologisch wird „El Shaddai“ mit einer ugaritisch-syrischen Berggottheit mit phallisch-kastrierendem Zerstörungspotenzial in Verbindung gebracht, stellt also in diesem Textzusammenhang passenderweise eine uralte Deifizierung eines phallischen Machtanspruchs dar. Das patriarchalische Primat als loyalitätserzeugendes und triebkontrollierendes Rechtsinstitut der sozialen Bezugsgruppe wird jedenfalls mittels einer Kastrationsandrohung etabliert. Einige Bibelverse weiter droht dieser Gott mit der ultimativen Kastration: Wenn du dich nicht an meine Regeln hältst, rotte ich dich aus. 
Man könnte die ganze Szene hinsichtlich der sadistischen Aspekte des Cottesbildes in etwa so paraphrasieren:

„Schau her Gott. Ich fürchte, dass Du mir eigentlich alles abschneiden willst. Ich habe ja auch schon gesehen, dass das wirklich bei manchen Männern so gemacht wurde. Deshalb mache ich mich jetzt selbst ganz klein und gebe Dir schon einmal ein (nicht ganz so wichtiges) Stück meines Gliedes. Dann lässt Du mir den Rest und damit darf ich mich zu Deinem Ruhme fortpflanzen. Ansonsten halte ich mich immer ganz genau an Deine Vorschriften, denn wenn ich mich nicht an sie halte, schneidest Du mir den Rest doch noch ab. Damit ich das nievergesse, macheich das auch mit meinen Söhnen. Die werden dann sehen, dass sogar ihr Vater Angst vor Dir hatte, weil er ja auch beschnitten ist. Und deshalb werden sie später auch Angst vor Deiner Macht haben und sich an Deine Gesetze halten."

Ich möchte meine Sichtweise auf die kulturhistorische Ableitung rituell gebundener Ängste und Aggression noch etwas erläutern. Frühmenschliche und vorgeschichtliche Gemeinschaften nutzten Rituale als magischen Versuch, um dem Horror existenzieller Bedrohungen durch unbeherrschbare, unverständliche und passiv erlittene Naturerscheinungen, Krankheit und Tod zu begegnen. Das mit diesen Bedrohungen und Katastrophen einhergehende Grauen soll durch die Wendung des Schreckens in einen Modus aktiven rituellen Gestaltens bewältigt, eingegrenzt oder zumindest erträglicher werden (Burkert 1997; Türcke 2012). Die Universalität des rituellen Menschenopfers (Rind 1998) in allen menschlichen Gemeinschaften verdeutlicht dies eindrücklich:

„Nicht ich kann jederzeit sterben oder in Stücke gerissen werden, sondern ich schwinge mich im Ritual zum Herr über Leben und Tod auf und besänftige gleichzeitig den unergründlichen Zorn der Natur und der Götter indem ich sie mit Opfern füttere."

Die entlastende Verwandlung des realen Grauens in sakrale Grausamkeit ist der funktionale Kern dieses Rituals.

In Anlehnung an den Verhaltenswissenschaftler Norbert Bischof beginnt der Lebenszyklus eines Rituals aus areligiöser Sicht als vorsprachlich-intuitive Inszenierung eines unter bestimmten historischen Bedingungen zunächst funktionalen, triebregulierenden Organisators sozialer Bindungen und Übergänge (z.B. patriarchalische Beschneidung Heranwachsender zur Triebkontrolle in einer prähistorischen Stammeskultur). Derartige initial adaptive gruppale Verhaltensmuster werden im Weiteren in einem normativen und legitimierenden Mythos verdichtet. Z.B. schließt ein Urvater einen Pakt mit der Gottheit, die bei Wohlverhalten beschenkt, bei Ungehorsam grausam bestraft: Sexualität und Fortpflanzung ja - aber nur unter dem strafbewehrten Primat patriarchalischer Triebkontrolle mit der impliziten Botschaft: Dein Glied gehört nicht dir. Und: Der Stärkere darf im Namen Gottes anderen Menschen Körperteile abschneiden. Basierend auf diesem Mythos kommt es schließlich zur dogmatischen und sanktionsbewehrten Ausformulierung eines dann 
kaum noch hinterfragbaren göttlichen Gebotes, das vermittelt in einem Ritual die Abgrenzung gruppaler Identität und die Ausübung klerikaler Macht ermöglicht. Jahrtausende später wird es als aus der Zeit gefallenes Relikt wie ein kulturgeschichtliches Treibgut oft nur noch aus unverstandener Loyalität heraus „geglaubt“ und, „weil es schon immer so war“ - im Fall der Beschneidung auf Kosten der betroffenen Jungen -, wiederholt.

Ein solcher Zyklus kann sich über sehr lange historische Zeiträume etablieren. Zwei durch gruppalen Konformitätsdruck vermittelte Mechanismen halten die wiederholende Reinszenierung des Rituals transgenerational aufrecht. Zum einen die durch das eigene Beschneidungstrauma bewirkte Wahrnehmungsstörung der elterlichen Täter gegenüber den Schmerzen, Ängsten und Gefahren ihrer Kinder sowie die folgende unbewusste Identifikation der Kinder mit dem elterlichen Aggressor:

„Es kann und darf nicht schlecht gewesen sein, was meine Eltern mit mir gemacht haben. Deshalb tue ich es auch. Und wenn ich es meinen Kindern dann auch angetan habe, kann ich nicht mehr zurück und einsehen, dass das unter anderem auch ein schlimmer Gewaltakt war.“

Aufgrund der hohen Eigenbetroffenheit der Ritualausübenden und der religiösen Referenzialität des Rituals entziehen sich diese Zusammenhänge häufig einer argumentativ-rationalen Auseinandersetzung.

Nachdenklichkeit und Änderungsbereitschaft kann am ehesten von innen her erfolgen, wenn die fehlende rationale Basis eines klerikal durchgesetzten, körper- und seelenverletzenden religiösen Rituals aufgrund von Widersprüchen zwischen einem sadistischen Gebot oder Gottesbild und einer zunehmend gewaltärmeren gesellschaftlichen Wirklichkeit mit der Zeit offensichtlich wird. Dies nehmen im Zusammenhang mit der rituellen Beschneidung offensichtlich zunehmend auch Betroffene innerhalb der Religionsgemeinschaften so wahr (Sadeh 2012; Enosch 2012; Segal 2012). Enosch spricht von der Beschneidung als einem Akt der Vergewaltigung, Segal nennt sie einen barbarischen Akt.

Anthropologische und kulturhistorische Betrachtungen zur Genese und möglichen Funktion des archaischen Beschneidungsrituals sind zu trennen von der modernen, neuzeitlich entwickelten empathischen Perspektive, die einzunehmen ist, wenn es um die möglichen Folgen der rituellen Beschneidung für das Erleben und die Entwicklung der heute betroffenen kleinen Jungen und Babys geht. Die aufgrund eigener aversiver kindheitlicher Erfahrungen unbewusst verinnerlichte Teilhabe an kollektiv verbindlichen Überzeugungen und an deren ritueller Ausgestaltung kann die empathische Einfühlung in das Erleben Anderer beeinträchtigen. Dies gilt für den (z.B. entwertenden oder territorial exklusiven) Umgang mit kulturell oder religiös andersartig organisierten Gruppen und sogar für die Affektzustände der eigenen Kinder. Die Praktizierung einer hochsanktionierten Gruppennorm in Form einer ri- 
tuellen Praxis kann daher auch eine Beeinträchtigung der intuitiv-empathischen Elternfunktionen bewirken, insbesondere dann, wenn die persönlichen und kollektiven Überzeugungen referenziell für Kohäsion und Identität der sozialen Bezugsgruppe sind und entsprechend über Tabus abgesichert bzw. geheiligt werden. Die destruktiven Exzesse aggressiver Ideologien oder die langwierige Entwicklung der empathischen Perspektive im Umgang mit Kindern, ausgehend von der Kindesopferung über die weit verbreitete Praxis der Aussetzung und der Prügelstrafe bis hin schließlich zur Formulierung der Kinderrechte, geben hierfür bedrückende Beispiele (DeMause 1980, 1994; Pinker 2011). Kollektive Überzeugungen werden gruppal besonders dann unreflektiert tradiert, wenn sich das rituell mithandelnde Individuum den konstitutiven Gruppennormen hierdurch verbunden fühlt und aufgrund in seiner persönlichen Kindheitsbiografie selbst erfahrener traumatischer (Ritual-) Handlungen den Gewaltaspekt des betreffenden Rituals aus eigenen Abwehrbedürfnissen und Loyalität zu den eigenen Eltern heraus verleugnen muss. Dann kann das emotionale Erleben von Angst, Schmerz und Schrecken des (z.B. auch kindlichen) Opfers des Rituals von den handelnden Erwachsenen nicht mehr empathisch erfasst werden und eine rituelle Täter-Opfer-Kette kann sich transgenerational über lange Zeiträume hinweg etablieren. Dies kann insbesondere unter Bedingungen, unter denen sich eine Gruppe in ihrer Existenz infrage gestellt oder sogar bedroht fühlen muss, zu noch stärkerer Identifikation mit diesen Überzeugungen und den zugehörigen Normen und Ritualen führen. Ein Leitmotiv für das ärztliche Handeln, das auf wissenschaftlicher Crundlage allein dem Wohl des individuellen Patienten verpflichtet ist und zu allererst Schaden von diesem abzuwenden hat, ergibt sich aus derartigen Zusammenhängen allerdings nicht. Rituell sanktionierte invasive Maßnahmen, insbesondere gegenüber nicht einwilligungsfähigen Personen, und ärztliches Handeln schließen sich daher per se aus.

\subsection{Ausprägungen und gängige Formen}

Über unterschiedliche Begründungszusammenhänge lässt sich die männliche Vorhautbeschneidung in vorwiegend

- rituell-religiöse,

- hygienisch-ästhetische und

- medizinische Varianten unterteilen.

Aufgrund der identitätsstiftenden hohen emotionalen Bedeutsamkeit der sexuellen Sphäre für das Individuum wie für die kulturelle Bezugsgruppe existieren jedoch Übergänge und Simultanitäten zwischen diesen Motivationslagen. Ritualbeschneidungen haben auch medizinische und psychische Aspekte für die Betroffenen (z.B. aufgrund von Komplikationen) und medizinisch begründete Beschneidungen können auf nur scheinbar plausiblen 
Indikationen beruhen oder ebenfalls psychotraumatische Folgen nach sich ziehen. Darüber hinaus ist aus entwicklungspsychologischer und juristischer Sicht der biografische Zeitpunkt der Durchführung ein bedeutsames Einteilungskriterium.

Die aufgrund eines individuell bestehenden medizinischen Problems indizierte und elterlich in Ersatzvornahme gestattete, unter sterilen Bedingungen und Anästhesie chirurgisch durchgeführte Zirkumzision im Kindesalter ist zu unterscheiden von der mit religiösen Vorschriften und identitätssichernden kulturellen Traditionen begründeten und daher kollektiv verbindlichen rituellen Entfernung der Vorhaut im Säuglings- oder Kindesalter. Eine säkulare Analogie besteht in der mit präventiv-medizinischen Argumenten begründeten, besonders in den USA bei 65\% der männlichen Neugeborenen (Alanis u . Lucidi 2004) praktizierten Routinebeschneidung ohne Bestehen eines individuellen körperlichen Krankheitszustandes.

Dem gegenüber steht die aus unterschiedlichen Motiven (s.u.) eigenverantwortlich veranlasste Zirkumzision des selbstbetroffenen, juristisch einsichtsund einwilligungsfähigen erwachsenen Mannes.

Die medizinisch indizierte Genitalbeschneidung des Jungen und des Mannes wird heute in Deutschland unter Beachtung medizinischer Standards unter sterilen Operationsbedingungen, Analgesie und Anästhesie ärztlich-chirurgisch mittels unterschiedlicher Methoden (Skalpell, spezielle Klemmen) durchgeführt. Je nach Technik werden mehr oder weniger große Hautanteile des inneren und äußeren Vorhautblattes und des Frenulums erfasst, sodass die am Penisschaft hinter der Kranzfurche der Eichel verbleibende Haut mehr oder weniger stark gestrafft und die Ringnarbe unterschiedlich weit von der Kranzfurche entfernt ist. Die Wundränder werden zumeist mit selbstauflösendem Nahtmaterial adaptiert und heilen innerhalb von zwei bis drei Wochen ab. Für diese Zeit wird sexuell aktiven Jugendlichen und Männern eine Karenz, daran anschließend zunächst eine vorsichtige (Wieder-)Aufnahme des Geschlechtsverkehrs empfohlen. Die zur Vermeidung einer Beschneidung oder Zirkumzision durchgeführte Erweiterungsplastik der Vorhaut wird nur selten durchgeführt. Eine weitere Methode ist die sogenannte Plastibell-Methode (Abschnürung der Vorhaut mittels einer Kunststoff-Glocke über etwa zehn Tage hinweg).

Bei der religiös motivierten rituellen Neugeborenen- oder Jungenbeschneidung im Alter von etwa fünf bis sieben Jahren wird - sofern nicht medizinisch fachgerecht durch Ärzte - durch rituell zuständige und geschulte Fachpersonen allerdings häufig unter Verzicht auf sterile Bedingungen und Schmerzbetäubung die vor die Eichel gezogene ggf. abgeklemmte kindliche Vorhaut teils mit einem Messer (äußeres Blatt), teils auch noch durch Abreißen von Vorhautresten an der Kranzfurche (inneres Blatt) und zumeist ohne Vernähung der Wundränder entfernt. 


\subsection{Häufigkeiten}

In verschiedenen Religionsgemeinschaften ist die genitale Beschneidung für alle männlichen Neugeborenen (z.B. im Judentum) oder Jungen etwa im Einschulungsalter (z.B. im Islam) obligatorisch. Etwa ein Drittel der Männer weltweit sind Schätzungen zufolge beschnitten. Die nicht religiös motivierte routinemäßig durchgeführte kindliche Vorhautbeschneidung wird heute am häufigsten in den USA durchgeführt. Hier sind entsprechend - mit allerdings sinkender Tendenz - etwa 6o\% der erwachsenen Männer beschnitten, in Südkorea (etwa zehn Prozent der Neugeborenen werden hier beschnitten) sogar über 90\% (Alanis u. Lucidi 2004). In den meisten europäischen Ländern wird die kindliche und männliche Genitalbeschneidung außerhalb ritueller Bezüge deutlich seltener durchgeführt, möglicherweise weil hier aufgrund einer erhöhten Sensibilität und Empathie für die kindesseitige Erlebnisverarbeitung eines einschneidenden Genitaltraumas die routinemäßige Neugeborenenbeschneidung eher kritisch beurteilt wird und darüber hinaus Krankenversicherungen die Erstattung anfallender Kosten für medizinisch nicht indizierte Beschneidungen ablehnen.

\subsection{Psychische Aspekte: Motive der Klienten}

Der Begründungsdiskurs der Neugeborenenbeschneidung wie auch der Beschneidung älterer Jungen wird beherrscht von offensiv formulierten hygienisch-medizinischen oder - innerhalb von Glaubensgemeinschaften - religiösen Vorstellungen und dann ggf. noch juristisch untermauert mit dem grundgesetzlich verbrieften Recht auf freie Religionsausübung (vgl. hierzu die gegenläufigen Rechtsauffassungen von Putzke 2008a; Schreiber et al. 2009; Herzberg 2012). Der entsprechende Ritus wird in verschiedenen Glaubensgemeinschaften mit kollektiv verbindlichen religiösen Gesetzen und Traditionen begründet, welche auf die Absicherung der religiös-kulturellen Identität und ein jeweils gottgefälliges Verhalten zielen. Referenziell verbindliche Vorbilder sind dabei beschnittene oder vorhautlos geborene religiöse Erzväter (Abraham) oder Propheten (Mohammed). Denen, die diesen Vorbildern folgen und dem Beschneidungsritual unterworfen werden, werden außerordentliche Cratifikationen in Aussicht gestellt, wie z.B. die Zugehörigkeit zu einer besonderen göttlichen Bundesgemeinschaft oder jenseitige Freuden.

Demgegenüber ist der Schutzanspruch des Kindes vor jeglicher Misshandlung, Missbrauch und Vernachlässigung sowie die Achtung seiner körperlichen Würde und Integrität als ethischer Standard nicht nur juristisch, sondern auch wissenschaftlich gut begründet (Egle u. Hardt 2005). Man tut Kindern nicht weh. Die Entwicklung dieses sozialen Mems begann allerdings in der Neuzeit auch in Europa erst 1693 mit John Lockes Schrift „Gedanken über Erziehung“, führte über die Formulierung der ersten Kinderschutzgesetze gegen 
Ende des 19. Jahrhunderts in England (Pinker 2011) schließlich hin zur UNKinderrechtskonvention von 1990 und bemerkenswerterweise erst im Jahr 2000 auch in Deutschland zum gesetzlich verankerten Recht von Kindern auf eine gewaltfreie Erziehung ( $\$ 1631$ Abs. 2 BCB). Demnach sind körperliche Bestrafungen, seelische Verletzungen und andere entwürdigende Maßnahmen durch die Eltern unzulässig. Wir wissen heute aus zahlreichen Untersuchungen, dass Kinder keine Schmerzen erleiden sollten und dass Eltern und Erwachsene an den Genitalien von Kindern - von medizinisch unabweisbaren Notlagen abgesehen - nichts zu manipulieren haben.

Ähnlich wie bei anderen Gesundheitsrisiken, die mit dem männlichen Geschlecht assoziiert sind (z.B. die deutlich erhöhte Suizidrate oder die um Jahre verringerte Lebenserwartung), besteht auch in Bezug auf die leidvollen Aspekte der weit verbreiteten medizinisch nicht indizierten Genitalbeschneidung von Jungen eine deutlich verringerte öffentliche Aufmerksamkeit und Sensibilität. Eine Ursache für die Ausblendung des sexuellen Gewaltaspektes bei der Genitalbeschneidung von Jungen besteht in der verglichen mit den meisten Formen der weiblichen Genitalbeschneidung objektiv weitaus weniger verstümmelnden männlichen Zirkumzision. Das bedeutet jedoch nicht, dass nicht auch die Entfernung der Vorhaut und des Vorhautbändchens juristisch nicht einwilligungsfähiger Jungen im Säuglings- und Kindesalter zu erheblichen körperlichen, sexuellen oder psychotraumatischen Komplikationen und Leidenszuständen bis hinein in das Erwachsenenalter führen kann (Taddio et al. 1997; Boyle et al. 2002; Okeke et al. 2006; Muula et al. 2007; Weiss et al. 2010; Franz 2010; Frisch et al. 2011). Diese Problematik wird - nicht zuletzt aufgrund religiöser oder kultureller Tabus und damit verbundener möglicher Konflikte - von Ausnahmen abgesehen derzeit noch vorwiegend in Fachkreisen und nicht öffentlich diskutiert. Es existieren jedoch keine rationalen wissenschaftlichen - weder hygienische noch medizinisch-präventive - Begründungen dafür, die Durchführung der männlichen Genitalbeschneidung nicht auf ein Lebensalter zu verschieben, in dem der Betroffenen sich selbst, möglichst frei von kindlichen Ängsten, eine eigene Meinung bilden und selbst entscheiden kann, ob eine Genitalbeschneidung für ihn selbst infrage kommt oder auch nicht. Die derzeitige Diskussion eines Verbotes kosmetischer Operationen im Kindes- und Jugendalter tangiert die umrissene Fragestellung durchaus und weist möglicherweise auf eine wachsende öffentliche Sensibilität im Bereich des Kindesrechtes auf körperliche Unversehrtheit hin.

Häufig wird eine rituell-religiöse Genitalbeschneidung nicht bei Neugeborenen, sondern bei Jungen im Alter von fünf bis sieben Jahren durchgeführt. Im Gegensatz zum Säugling verfügt das Kind in dieser Entwicklungsstufe über differenziertere Wahrnehmungsmöglichkeiten für Beziehungen, Fakten und Vorgänge in seiner Umgebung. Andererseits ist es aber auch noch bestimmt von wahrnehmungsverzerrenden kindlich-triebhaften Fantasien und Ängsten, die es auf den empathischen Schutz durch erwachsene Bezugspersonen 
angewiesen sein lassen. Dies gilt grundsätzlich auch für das Erleben der eigenen Beschneidung auf dem Höhepunkt der ebenfalls auch noch phantasmatisch bestimmten Konsolidierungsphase der sexuellen Identitätsentwicklung des Jungen. Dabei könnte aus psychoanalytischer Sicht die rituelle Beschneidung des Cliedes kleiner Jungen im Alter von etwa fünf bis sieben Jahren eine kollektive, potenziell sexualtraumatische Erfahrung darstellen, die besonders in patriarchalisch geprägten Kulturen eine Ursache für die beobachtbare starke soziale Kontrolle im sexuellen Bereich (z.B. Geschlechtertrennung, Ehestiftungen, starke Sanktionierung des Ehebruchs) und der Kontrolle der Frau (Verhüllung zur Vermeidung aufreizenden Verhaltens, tendenzielle Beschränkung auf den häuslichen Bereich, Beaufsichtigung durch männliche Verwandte) sein könnte. Die potenziell traumatische Wirkung dieses Rituals mag in patriarchalischen Gesellschaften eine Stabilisierung der Machtstrukturen und familialen Bezüge bewirken. Aus psychoanalytischer Sicht resultiert aus dieser definitiven und traumatischen Klarstellung hierarchischer Bezüge, die mit der im Erleben vieler Kinder sehr realen Möglichkeit einer Kastration einhergeht, eine durch starke Ängste fundierte patriarchalische Loyalität.

Die genitale Beschneidung des Jungen auf dem Höhepunkt der infantilen Sexualentwicklung bringt jedenfalls besondere Entwicklungsrisiken mit sich. Die Beschneidung kann von Jungen, die sich in dieser Phase zunehmend auf ihre Genitalität und männliche Rolle zentriert erleben, wie eine schwere Sanktions- oder Kastrationsdrohung erlebt werden. Der schmerzlich-traumatische Eingriff erfolgt unverstellt, bewusst wahrnehmbar und unter direktem Zugriff auf den libidinös und narzisstisch hoch besetzten Genitalbereich. Der ängstigende Gewaltaspekt unterliegt dabei einer bemerkenswerten Verleugnung durch die beteiligten Erwachsenen. Er wird rationalisiert als festlich und freudig gestalteter Männlichkeitsritus z.B. durch die Ausstaffierung des kleinen Jungen (der ja noch in keiner Weise an der Schwelle zum Mannesalter steht) mit hypermaskulinen Attributen, entsprechenden Kostümierungen und großen Geschenken.

Die Gewalt des Beschneidungsrituals besteht aber gleichwohl in einer schweren irreversiblen Körperverletzung der Intimzone, in der Inkaufnahme eines erheblichen Risikos für physische und psychische Komplikationen und Langzeitrisiken bis hin zu einer beeinträchtigten sexuellen Empfindungsfähigkeit. Und das alles ohne Einwilligung des hiervon hilflos betroffenen und in seinem eigenen Recht auf Religionsfreiheit beeinträchtigten Kindes. Die empirischen Arbeiten hierzu liegen vor (vgl. Franz 2012; Frisch et al. 2011). Eine die Säuglingsbeschneidung befürwortende Stellungnahme der Autoren der American Academy of Pediatrics im sogenannten „Circumcision Policy Statement“ und im zugehörigen „Technical report“ mit dem Titel „Male Circumcision“ von 2012 wird wegen Befangenheit, erheblicher Interessenkonflikte, einer völlig unzureichenden Methodik sowie eines massiven Reportbias auch von deutschen ärztlichen Fachverbänden kritisiert (vgl. auch www. 
doctorsopposingcircumcision.org oder www.circumcision.org/aap.htm). Ein Mitglied des Autorenteams - Douglas Diekema - empfahl in anderen Zusammenhängen sogar die Inzision weiblicher Genitalien (Diekema 2010). Andrew Freedman, ein weiterer Autor des Statements, hat mitgeteilt, dass er selbst seinen eigenen Sohn aus rituellen Gründen auf dem Küchentisch der Eltern beschnitten habe. Er liefert einen Hinweis auf den starken traditionalen Gruppendruck in seiner Begründung für diesen Akt:

"I circumcised him myself on my parents' kitchen table on the eighth day of his life. But I did it for religious, not medical reasons. I did it because I had 3,00o years of ancestors looking over my shoulder" (Freedman 2012).

Die Verleugnung des Gewaltaspekts der Genitalbeschneidung kleiner Jungen ist bei einer so großen zuschauenden Ahnenreihe in ihren Ursachen vielleicht teilweise nachvollziehbar, muss aber wegen der möglichen erheblichen Komplikationen gleichwohl thematisiert werden. Ein besonders eklatantes Beispiel für diese Verleugnung gegen jede wissenschaftliche Evidenz in Bezug auf das Schmerzerleben des Neugeborenen stellt die Homepage von Rabbi Goldberg (www.beschneidung-mohel.de/ablauf_und_heilungsprozess.html) dar:

„Findet die Beschneidung am 8. Tag statt, wird kein Betäubungsmittel injiziert. Betäubungsmittel die injiziert (gespritzt werden) tragen ein weit höheres Risiko als der Schmerz durch den Schnitt. Der Schmerz ist bei kleinen Babys minimal, weil das Schmerzempfinden noch nicht voll ausgebildet ist."

Das Betrachten eines Beschneidungsvideos kann jeden Beobachter leicht vom Gegenteil überzeugen (www.youtube.com/watch?v=Q3w5QlKV6gA).

Nicht zuletzt besteht die vom Beschneidungsritual ausgehende Gewalt auch in der verstörenden Gewaltzeugenschaft, der viele Kinder in den unterschiedlichen Beschneidungskulturen im Laufe ihrer Kindheit immer wieder ausgesetzt werden, wenn sie als Zeugen den rituellen Gewaltakt - ausgeführt oder gestattet von den elterlichen Bezugspersonen - erleben und bewältigen müssen. Manche Betroffene erleben und schildern dies im Nachhinein wie einen schweren Vertrauensbruch in der Beziehung zu ihren Eltern.

Die Motivationslagen volljähriger und einwilligungsfähiger Männer, sich für eine Zirkumzision zu entscheiden, betreffen (z.B. bei Konvertiten) ebenfalls rituell-religiöse Aspekte wie auch medizinische Indikationen, beruhen aber auch auf persönlichen ästhetischen Überzeugungen oder kosmetischen Standards der Bezugsgruppe sowie auf sexuellen oder hygienischen Präferenzen. Diese müssen im Einzelnen natürlich nicht mit medizinischen Begründungen assoziiert sein. Bewusst oder unbewusst kann neben den genannten Motiven auch der Wunsch nach körperbezogenen Manipulationen (in Analogie zum Piercing oder zur Tätowierung) entschlussbildend mitwirken. 


\subsection{Medizinische Aspekte: Indikation, Nebenwirkungen, Wechselwirkungen}

Aus medizinischen Gründen ist bei etwa vier Prozent aller Jungen eine Zirkumzision indiziert. Indikationen zur Zirkumzision sind bis ins Vorschulalter persistierende, nicht ohne weiteres lösbare Vorhautverklebungen, chronische oder rezidivierende Balanoposthitiden, Balanitis xerotica obliterans, Lichen sclerosus der Vorhaut, schmerzhafte oder dysfunktional behindernde Vorhautverengungen und hierdurch bedingten Miktionsstörungen und lokale Entzündungen sowie Harnwegsinfekte auf der Grundlage von gravierenden urologischen Erkrankungen der vorgeordneten Harnwege (nach Stehr et al. 2001). Zusätzlich zu einer medizinischen Behandlungsindikation muss nach vollständiger Aufklärung eine Einwilligung entweder des juristisch einsichts- und einwilligungsfähigen Betroffenen selbst oder der elterlichen Sorgeberechtigten vorliegen.

Die rituelle Genitalbeschneidung von Jungen ist aus der Sicht vieler säkularer Kritikerinnen und Kritiker ein schmerzhafter und gefährlicher Gewaltakt mit negativen gesundheitlichen, sexuellen und seelischen Komplikationen für viele der Betroffenen. Es existiert keine medizinische Begründung dafür, kleinen Jungen ohne deren Einwilligung ihre gesunde Vorhaut abzuschneiden.

Mögliche lokale Komplikationen der Vorhautbeschneidung sind Nachblutungen, eine postoperative Narbenstenose an der belassenen Restvorhaut, venöse Knoten der Penishaut, Verwachsungsstränge zwischen der Eichel und der anschließenden Haut des Penisschaftes mit dem Risiko von Erektionsbehinderungen und Entzündungen und der Notwendigkeit weiterer operativer Revision, Erektionsbehinderung durch ein verkürztes Frenulum, versunkener Penisschaft durch zu ausgiebige Resektion der Penisschafthaut, lokale Wundheilungsstörungen mit Gewebeverlust bis hin zur Gangrän, Wundinfektionen, Sepsis, unbeabsichtigte (Teil-)Amputationen der Eichel oder des Penisschaftes, Verengungen der Harnröhrenmündung an der Eichel (besonders häufig nach der Neugeborenenbeschneidung), Harnröhren-Fistelbildung, sich entwickelnde sexuelle Sensibilitätsverluste im Bereich der Eichel und aufgrund des Verlustes des hochsensiblen Vorhautgewebes. Die Häufigkeit postoperativer Komplikationen unter Beachtung medizinischer Standards wird mit etwa zwei Prozent der Fälle angegeben (Stehr et al. 2001). Anderen Untersuchungen zufolge schwanken die Komplikationsraten zwischen Null und 16\% in Abhängigkeit von der Erfahrung und den professionellen Bedingungen (Weiss et al. 2010) bis hin zu 24\% (Muula 2007). Darüber hinaus bestehen Risiken bedingt durch Narkosezwischenfälle bis hin zu irreversiblen cerebralen Schäden und Tod. Schließlich sind abhängig vom kindlichen psychosexuellen Entwicklungsstadium, in dem der Eingriff vorgenommen wird, auch bleibende psychotraumatische Folgen bis ins Erwachsenenalter möglich, je nachdem unter welchen traumatischen Umständen für das Kind beispielsweise die ri- 
tuell geforderte Beschneidung erfolgte. Dies gilt im Falle der Neugeborenenbeschneidung im Sinne einer Intensivierung des postnatalen Adaptationsstresses durch Schmerzen und aversiv-artifizielle Manipulationen mit entsprechenden Abstimmungsstörungen im Bindungsumfeld des Babys. Im Fall der rituellen Beschneidung fünf- bis siebenjähriger Jungen kann wie oben dargestellt die durch phantasmatische Ängste verzerrte kindliche Situationswahrnehmung und -verarbeitung der Genitalbeschneidung eine angstfreie Konsolidierung der sexuellen Identität und Körperlichkeit auf Dauer erschweren (Taddio et al. 1997; Ramos u. Boyle 2001; Boyle et al. 2002; Fallbeispiele bei Kelek 2006; Franz 2010).

Es existieren darüber hinaus eine Fülle rechtfertigender, zum Teil pseudorationaler oder scheinmedizinischer Begründungen der Kindesbeschneidung, die sich auf hygienische oder andere gesundheitliche Risiken beziehen. Dabei ist bemerkenswert, dass die von Erwachsenen aufgebrachte Akribie, Energie und Kreativität bei der Findung von medizinischen Vorteilen auch der rituellen Frühbeschneidung (heute überholte medizinische Argumentationsfiguren bezogen sich auf die gesundheitsförderliche Verhütung von Masturbation, Hysterie oder Epilepsie) die Bemühungen zur Einfühlung in kindliche Erlebensweisen angesichts drohender Verletzungen des Intimbereiches bei Weitem zu übersteigen scheint. Angeführt werden die Vorbeugung von (sehr seltenen) Entzündungen der kindlichen Harnwege und von (noch selteneren und spät auftretenden) Peniskarzinomen aufgrund besserer hygienischer Verhältnisse nach Zirkumzision, wobei heutige Standards eine effektive Genitalhygiene auch ohne operativen Eingriff ermöglichen und die Erkrankungswahrscheinlichkeiten so gering sind, dass sie unterhalb der Komplikationsrate des Bescheidungseingriffs liegen (Stehr et al. 2001). Weiterhin wird die durch die Zirkumzision möglicherweise erschwerte Übertragung sexuell vermittelter Infektionen (z.B. HIV, Herpesviren, Syphilis, Gonorrhoe oder humane Papillomviren) angeführt. Diese Post-hoc-Rationalisierungen der rituellen Kindesbeschneidung waren früher zur Begründung nicht möglich, zudem besitzen sie häufig keine wirklich abgesicherte empirische Basis (Stehr et al. 2001; Alanis u. Lucidi 2004). Wahrscheinlich besteht eine infektionsprophylaktische Wirkung der Zirkumzision bezüglich ulcerierender Genitalinfektionen (Alanis u. Lucidi 2004; Gray et al. 2009) und hinsichtlich pathogener HPV-Varianten (Tobian et al. 2009; Gray et al. 2010; Serwadda et al. 2010; Viscidi u. Shah 2010) sowie am besten gesichert für HIV in Hochrisikogruppen (Siegfried et al. 2009). Als ursächlich hierfür werden eine hohe Suszeptibilität der CD4-Rezeptoren der Langerhans Zellen des inneren Vorhautblattes für HI-Viren sowie das feuchte Präputialmilieu und dortige Mikroläsionen angenommen.

Aktuell kann auf drei große Bevölkerungsstudien zur HIV-Prophylaxe mittels Zirkumzision hingewiesen werden, die prospektiv, kontrolliert und randomisiert an jugendlichen und erwachsenen heterosexuell orientierten Männern aus Südafrika, Uganda und Kenia durchgeführt wurden (Siegfried et al. 2009; 
Auvert et al. 2005; Bailey et al. 2007; Gray et al. 2007). Es zeigte sich eine bedeutsame relative Reduktion der Rate der Neuinfektionen zwischen 38 und $66 \%$ innerhalb von zwei Jahren in der Interventionsgruppe im Vergleich zur unbeschnittenen Kontrollgruppe. Die Komplikationsrate der Zirkumzision lag zwischen 1,7 und 7,6\%. Allerdings können diese Studien argumentativ nicht zur Rechtfertigung einer generellen oder rituellen Beschneidung herangezogen werden, da fünf- bis siebenjährige Jungen oder gar Neugeborene sexuell noch nicht aktiv sind und das Alter der jüngsten in die Stichprobe eingeschlossenen Jugendlichen bei 15 Jahren lag. Außerdem kann diese ökonomisch sicher günstige Maßnahme nicht als alleinige hinreichend wirksame Präventionsmaßnahme zur Vorbeugung von HIV-Infektionen angesehen werden (Hallett et al. 2011). Präservative beispielsweise haben eine wesentlich bessere Schutzwirkung gegen sexuell übertragbare Infektionserkrankungen.

\subsection{Rechtliche Situation}

Die Verschwiegenheit oder das rationalisierende Herunterspielen möglicher körperlicher und seelischer Schadensfolgen ist im Hinblick auf die Beschneidung der weiblichen Genitalien heute durchbrochen worden. Während die beispielsweise in vielen afrikanischen Ländern nach wie vor weit verbreitete Praxis der verstümmelnden weiblichen Genitalbeschneidung mittlerweile $z u$ internationaler Ablehnung durch die WHO und u.a. auch in Deutschland zu strafrechtlicher Sanktionierung (z.B. auf der Grundlage von $\mathbb{} 223$ in Verbindung mit $\$ 224$ Abs. 1 Nr. 2 StGB bezogen auf die durchführenden Personen oder auf der Grundlage von $\$ 225$ StGB bezogen auf die Eltern) geführt hat, ist dies bei der männlichen Genitalbeschneidung nicht der Fall.

Das Alter des Jungen, ab dem von einer Einwilligungsfähigkeit und Einsichtsfähigkeit in die Tragweite des Eingriffs und dessen Irreversibilität und Risiken ausgegangen werden kann, ist in Deutschland umstritten. Es existiert ein Gerichtsurteil des Landgerichtes Frankenthal aus 2004, das einem neunjährigen Kind eine entsprechende Einwilligungsfähigkeit abspricht, eine andere Entscheidung des OLG Frankfurt a.M. aus 2007 orientiert sich am Alter der Religionsmündigkeit von 12 Jahren (nach $\mathbb{s} 5$ des RelKErzG kann ein Kind ab einem Alter von 12 Jahren nicht gegen seinen Willen in einem anderen Bekenntnis als bisher erzogen werden) als Kriterium der Einwilligungsfähigkeit. Diese Rechtsauffassung wird jedoch von Putzke (2008b) und Schreiber et al. (2009) kritisch kommentiert, da die prinzipiell reversible Entscheidung für eine bestimmte religiöse Bindung nicht mit der Entscheidung für eine irreversible Verletzung der körperlichen Integrität zu vergleichen sei. Außerdem sei auch im Alter von 12 Jahren nicht sichergestellt, dass eine Entscheidung des Kindes für eine Beschneidung wirklich autonom an eigenen Interessen orientiert ist und nicht elterliche Überzeugungen und Forderungen aufgrund kindlicher Loyalität zu den Bezugspersonen lediglich formelhaft als eigener Ent- 
schluss ausgegeben würden. Nach diesen Autoren ist eine rituelle Zirkumzision nur mit Einwilligung des zu Beschneidenden rechtlich zulässig, sie sehen eine Einwilligungsfähigkeit nicht vor Vollendung des 16. Lebensjahres gegeben und empfehlen eine Einholung der Einwilligung des Jugendlichen nach ausführlicher Aufklärung in einem ärztlichen Gespräch getrennt von den Eltern. Sie warnen vor möglicherweise zunehmenden Schadensersatzprozessen von als Kindern beschnittenen Erwachsenen, die mit dem Resultat der Beschneidung nicht einverstanden sind, vorgetragen gegen die ehemalig handelnden Ärzte oder auch Eltern. Eine Zwischenstellung nimmt Herzberg (2012) ein. In Analogie zur ab einem Alter von 14 Jahren grundsätzlich gegebenen strafrechtlichen Verantwortlichkeit z.B. für eine schuldhaft an einer anderen Person begangenen Körperverletzung (nach $\mathbb{\$} 19$ StCB, 3 JCG) folgert er ab diesem Alter eine Verantwortlichkeit oder Verantwortungsfähigkeit auch in Bezug auf die Durchführung einer Verletzung des eigenen Körpers. Herzberg stützt sich bei dieser Argumentation hilfsweise auch auf $₫ 5$ des RelKErzG, da ein Kind ab einem Alter von 14 Jahren sich selbst für die Zugehörigkeit zu einem religiösen Bekenntnis entscheiden kann. Kritisch räumt der Autor allerdings ein, dass es nicht zwingend sei, die Rechtswirksamkeit dieser Entscheidung auch auf ein Verletzungsritual zu erstrecken, das mit dem gewählten Bekenntnis eng verbunden ist. Schreiber et al. (2009) erwähnen einen Fall, bei dem ein Vater ohne Kenntnis der allein sorgeberechtigten Mutter seinen 12-jährigen Sohn beschneiden ließ und dieser dann ein erhebliches Schmerzensgeld erfolgreich von seinem Vater einklagte. Putzke (2008a) nimmt in ähnlicher Weise Stellung:
„Wer eine Zirkumzision an einem Kind vornimmt (gleichgültig, ob es sich etwa um einen Arzt, einen Mohel oder Sünnetci handelt), braucht, um die Verletzung des Kör- pers gerechtfertigt vorzunehmen, eine wirksame Einwilligung der Personensorgebe- rechtigten. Sie ist nur dann wirksam, wenn über das betroffene Rechtsgut disponiert werden darf, wobei das, Wohl des Kindes' ausschlaggebendes Kriterium ist. Nicht im ,Wohl des Kindes' liegt eine Zirkumzision, wenn sie medizinisch nicht notwendig ist. Dazu zählen nicht nur hygienisch und ästhetisch motivierte Zirkumzisionen, sondern auch religiöse Beschneidungen. Geht jemand irrig davon aus, dass die Einwilligung des oder der Personensorgeberechtigten rechtfertigend wirkt, so handelt er zumindest ohne Schuld - bei Unvermeidbarkeit des Irrtums. Dass Operateure bislang einen sol- chen Irrtum vermeiden konnten, ist fraglich. Deshalb ist strafrechtliche Schuld nicht in der Vergangenheit zu suchen. Für die Zukunft wird man allerdings von der Vermeid- barkeit ausgehen müssen [...] Wer einen Minderjährigen ohne medizinische Indikation zirkumzidiert, wenn etwa allein hygienische, ästhetische oder religiöse Gründe vor- liegen, macht sich strafbar nach $\$ 223$ StCB.“"

Aus juristischer wie auch aus ärztlicher Sicht stellt die rituelle Genitalbeschneidung bei Fehlen einer medizinischen Behandlungsindikation und fehlender Einsichts- und Einwilligungsfähigkeit des Kindes sowie bei bezüglich der Verletzung der körperlichen Integrität des Kindes nicht gegebener elter- 
licher Verfügungsberechtigung grundsätzlich also einen körperverletzenden Eingriff dar ( $\$ 223$ StCB). Bezogen auf die emotional hochbesetzte sexuell-intime Sphäre des Kindes ist er verbunden mit einer bleibenden Verletzung der körperlichen Integrität und der psychosexuellen Identität sowie entsprechenden Haftungsrisiken (Schreiber et al. 2009; Stehr et al. 2008).

Diese Sichtweise wurde durch ein Urteil des Kölner Amtsgerichtes im Wesentlichen nachvollzogen. Grundlage war eine auf Wunsch der Eltern durchgeführte, islamisch-religiös motivierte Beschneidung eines vierjährigen Jungen im November 2010, bei dem es trotz fachlich korrekter Ausführung durch einen niedergelassenen Arzt zu erheblichen Blutungskomplikationen und Nachoperationen kam. Das Amtsgericht Köln sprach den angeklagten Arzt aufgrund eines unvermeidbaren Verbotsirrtums ( $\mathbb{1 7} \mathrm{StCB})$ zwar frei. Das zuständige Landgericht sah nach staatsanwaltlich eingelegter Berufung in seinem Urteil vom 7. Mai 2012 den Tatbestand der einfachen Körperverletzung jedoch als erfüllt an, da der Eingriff nicht dem Kindeswohl und dem Recht des Kindes auf körperliche Unversehrtheit entspreche.

In der Folge kam es erstmals in Deutschland zu teilweise sehr polarisierend und polemisch, wenig argumentativ geführten Diskussionen, obwohl bis dahin öffentlich weithin eine bemerkenswerte Indifferenz in der ethischen Beurteilung der männlichen Genitalbeschneidung im Kindesalter bestand. Die Heftigkeit der Debatte um die männliche Genitalbeschneidung lässt dabei auf tiefgreifende Ängste und Konflikte schließen. Dies geschah vor dem Hintergrund, dass in der jüdischen und islamischen Kultur starke Ängste bestehen, auf die Beschneidung zu verzichten. Andererseits befürchteten viele säkulare Demokraten die Beschädigung einer freiheitlichen staatlichen Grundordnung durch anachronistische religiöse Machtansprüche.

Die Beschneidung ist aus Sicht der Praktizierenden eine im transzendentalen wie im gruppalen Sinn identitätsstiftende Referenz, kein Trauma, sondern Vervollkommnung. Speziell aus jüdischer Sicht ist sie für viele Betroffene sogar noch mehr. Sie ist angesichts ungeheurer Verbrechen an jüdischen Menschen für viele ein Überlebenszeichen. Ein Beweis für ein Weiterleben trotz umfassender Katastrophen. Dass aus jüdischer Sicht eine Kritik an der Beschneidung gerade auch in Deutschland angesichts der historischen Verstrickungen in Schuld und Verantwortung tatsächlich wie eine erneute Bedrohung erlebt werden kann, ist insofern nachvollziehbar. Religiöse Verteidiger des Beschneidungsrituales müssen aber heute akzeptieren, dass sie keine exklusive Deutungshoheit über das von ihnen praktizierte Beschneidungsritual und dessen traumatische Aspekte mehr haben. Auch nicht in Deutschland.

Am Thema der rituellen Beschneidung von kleinen Jungen kondensiert sich zum einen ein elementarer Wertekonflikt zwischen anscheinend nicht verhandelbarer Beschneidungspraxis verschiedener religiöser Gruppen und dem im Grundgesetz und der UN-Kinderschutzkonvention unteilbar gültigen säkularen Recht auf körperliche Unversehrtheit und dem Recht des Kindes vor 
gesundheitsschädlichen Bräuchen geschützt zu werden. Die Auseinandersetzung folgt dabei entlang der tektonischen Spannungslinien epochaler Trends zugunsten einer in Europa zunehmenden Gewaltfreiheit und Sensibilität für Kinderrechte. Sie wird zudem bestimmt durch Ängste um die eigene Identität und den Verlust gruppaler Kohärenz. Außerdem wird die Faktentreue der Wahrnehmung durch Ängste vor Kastration und existenzieller Finalität beeinträchtigt. Vor allem aber findet sie im Horizont antisemitischer Verstrickungen und des Holocaust und dem hierdurch bis heute verursachten Leid statt. Religionsvertreter sahen jüdisches und muslimisches Leben in Deutschland als bedroht oder sogar als unmöglich an, sollte die Beschneidung weiterhin als Körperverletzung strafbar sein. Aus diesen Gründen wurde die Beschneidungsdiskussion und ihre politische Instrumentalisierung nach dem Urteil des Kölner Landgerichtes weniger von Fakten als von religiös-dogmatischen Setzungen und Unterstellungen bestimmt, die nicht mehr so recht in die Diskurslandschaft einer säkularen Wissensgesellschaft passen.

Die entstandene Debatte fand zunächst ein vorläufiges Ende mit Inkrafttreten des neuen Beschneidungsgesetzes am 28. Dezember 2012. Dieses Gesetz erlaubt die Beschneidung von Jungen in Deutschland auch ohne medizinische Indikation unter bestimmten - oder besser - unter relativ unbestimmten Bedingungen. Beispielsweise wurde die medizinische Fachkunde des Durchführenden relativiert, die Frage der Analgesie/Anästhesie blieb unklar und Jungen können auf Wunsch der Eltern auch aus anderen Gründen als religiösen sowie gegen ihren offensichtlichen Willen beschnitten werden. Die unpräzisen Formulierungen des Gesetzes hinsichtlich der Beschneidungsmotive könnten sogar zu Konstellationen führen, in welchen es Müttern oder Vätern, die beispielsweise die Selbstbefriedigung ihres Jungen unterbinden möchten, verboten wäre, ihren Jungen deswegen zu schlagen. Es wäre ihnen aber erlaubt, den Jungen beschneiden zu lassen. Strafrechtler wie Holm Putzke sehen durch die jetzige Regelung wesentliche Grundrechte wie das auf körperliche Unversehrtheit, Selbstbestimmung, Religionsfreiheit und den Gleichheitsgrundsatz verletzt (Eckert 2013). Das vom Deutschen Bundestag verabschiedete, in sich höchst widersprüchliche Gesetz zur Jungenbeschneidung wird die Debatte voraussichtlich nicht beenden. Es wird vielleicht sogar Wünschen nach Erlaubnis der rituellen Genitalbeschneidung auch von Mädchen Vorschub leisten. Möglicherweise werden erst sehr hohe Schadensersatzforderungen Betroffener zu einem Umdenken führen.

Es bleibt zu hoffen, dass mit einer wachsenden Bereitschaft zur empathischen Einfühlung in das Erleben der betroffenen Kinder auch die Sensibilität für die mit dem Eingriff verbundenen Risiken zunimmt und überkommene Rituale und Bräuche mit zunehmend kritischem Bewusstsein auf ihre heutige Angemessenheit reflektiert werden können. Die zeitliche Verschiebung der rituellen Beschneidung bis zur Einwilligungsfähigkeit des Jungen oder die symbolische Transformation der traumatischen Aspekte des Rituals wie z.B. im Brith 
Shalom wären wirklich am Kindeswohl orientierte Alternativen. Dazu bräuchte es allerdings mutige Erwachsene.

Demgegenüber ist die fachgerecht ärztlich durchgeführte Zirkumzision bei einem erwachsenen, einwilligungsfähigen Mann aus medizinischen Gründen aber auch aufgrund persönlicher ästhetischer, sexueller, hygienischer oder ritueller Präferenzen nach vollständiger Aufklärung über mögliche Risiken aus rechtlicher Sicht unproblematisch. Eine Erstattung der für die Durchführung des ärztlichen Eingriffs anfallenden Kosten durch die Krankenkassen erfolgt jedoch nur bei Vorliegen einer medizinisch begründeten Operationsindikation. Es ist jedoch aus psychotherapeutischer Sicht empfehlenswert psychodiagnostische Expertise hinzuzuziehen, wenn patientenseitig Hinweise auf eine Persönlichkeitsstörung oder körperdysmorphophobe Befürchtungen bestehen oder konfliktneurotische und unbewusst selbstschädigende Tendenzen mitbestimmend für den Wunsch nach Körpermanipulationen im Genitalbereich (z.B. durch Beschneidung oder aber auch durch eine Vasektomie) sein könnten. Dies könnte dazu beitragen, möglichen postoperativen Verarbeitungsstörungen und (auto-)destruktiven Eskalationen vorzubeugen (Püschel u. Cordes 2001; Franz 2010).

\subsection{Fazit für den Umgang im klinischen Alltag}

Die Entfernung der Vorhaut des männlichen Gliedes mittels operativer Zirkumzision oder anderer Verfahren ist ein weltweit verbreiteter und seit Jahrtausenden durchgeführter verletzender Eingriff, der die körperliche Integrität der männlichen sexuellen Intimsphäre irreversibel schädigt. Juristisch ist die medizinische Beschneidung der Vorhaut des männlichen Gliedes daher einer Körperverletzung gleichzusetzen. Aus ärztlicher Sicht erforderlich ist zur Durchführung deshalb eine medizinische Indikation aufgrund eines zu dokumentierenden individuellen Krankheitszustandes, der nach erfolglosem Einsatz konservativer Behandlungsmethoden durch die Beschneidung - ohne künftige medizinische Nachteile für den Patienten aufgrund des Vorhautverlustes - mit überwiegender Wahrscheinlichkeit geheilt oder gebessert werden kann. Weiterhin erforderlich ist eine Einwilligung des Betroffenen nach ausführlicher Aufklärung bei gegebener Fähigkeit zur Einsicht in die Tragweite und potenziellen Risiken des Eingriffs. Der Eingriff soll auf der Grundlage guter klinischer Praxis unter sterilen operativen Bedingungen unter Analgesie, Anästhesie und Nachbeobachtung von einem fachlich versierten Arzt durchgeführt werden.

Handelt es sich bei dem erkrankten Patienten um einen minderjährigen Jungen, welcher altersentsprechend noch nicht einsichts- oder einwilligungsfähig ist, müssen die sorgeberechtigten Personen, zumeist beide Eltern, eine Einwilligung für den Eingriff erteilt haben. Bei einem jugendlichen Patienten ab zwölf Jahren, spätestens ab 16 Jahren sollte aber in jedem Falle zusätzlich 
zum Votum der Sorgeberechtigten ein Einwilligungsvotum des Patienten in einem getrennt von den Eltern stattfindenden Gespräch eingeholt und dokumentiert werden.

Dies alles gilt nicht für die Beschneidung eines gesunden nicht einwilligungsfähigen Jungen. Soll an einem nicht einsichtsfähigen minderjährigen Jungen ohne Vorliegen eines individuell bestehenden Krankheitszustandes, der durch eine Beschneidung geheilt oder gebessert werden könnte, auf Verlangen eines Sorgeberechtigten beispielsweise unter Angabe von religiösen Gründen eine medizinisch nicht indizierte rituelle Beschneidung vorgenommen werden, ist dies ärztlicherseits zwar abzulehnen, da eine solche Maßnahme nicht dem Kindeswohl entspricht. Dies gilt insbesondere, wenn das Kind Zeichen von Angst und Abwehr gegenüber der Beschneidung zeigt. Gleichwohl ist es aufgrund der derzeitigen Gesetzeslage in Deutschland aber gestattet die Beschneidung des Jungen auf Wunsch der Eltern vorzunehmen. Eine obligatorische staatsanwaltliche Strafverfolgung wie im Falle einer Beschneidung weiblicher Genitalien erfolgt derzeit in Deutschland nicht. Handelt es sich um einen mindestens 16-jährigen Jugendlichen, der mit der Durchführung einer medizinisch nicht indizierten Beschneidung sicher einverstanden ist, könnte die Durchführung des Eingriffs erwogen werden.

Bei einem rechtsmündigen, einsichts- und einwilligungsfähigen Erwachsenen, der aus anderen als medizinischen Gründen bei sich eine Beschneidung durchführen lassen will, ist ebenfalls eine umfängliche Aufklärung und die Einwilligung zu dokumentieren, bevor der Eingriff durchgeführt wird. Bei Verdacht auf selbstschädigende Tendenzen etwa vor dem Hintergrund einer schweren Persönlichkeitsstörung oder dysmorphophoben Ängsten sollte aufgrund von Haftungsrisiken zuvor eine psychodiagnostische Abklärung erfolgen. Die für einen Eingriff ohne medizinische Indikation entstehenden Kosten werden in Deutschland nicht von den Krankenkassen übernommen.

\section{Literatur}

Alanis MC, Lucidi RS (2004) Neonatal Circumcision: A Review of the World's Oldest and Most Controversial Operation. Obstetrical \& Gynecological Survey 59(5), 379-395

Auvert B, Taljaard D, Lagarde E, Sobngwi-Tambekou I, Sitta R, Puren A (2005) Randomized, controlled intervention trial of male circumcision for reduction of HIV infection risk: the ANRS 1265 Trial. PLoS Med 2(11), e298

Bailey RC, Moses S, Parker CB, Agot K, Maclean I, Krieger JN, Williams CF, Campbell RT, Ndinya-Achola J0 (2007) Male circumcision for HIV prevention in young men in Kisumu, Kenya: a randomised controlled trial. Lancet 369(9562), 643-656

Boyle GJ, Goldman R, Svoboda JS, Fernandez E (2002) Male circumcision: pain, trauma and psychosexual sequelae. Journal of Health Psychology 7(3), 329-343

Burkert W (1997) Homo necans: Interpretation altgriechischer Opferriten und Mythen. 2. Auflage. de Gruyter Berlin

DeMause L (1980) Hört ihr die Kinder weinen. Suhrkamp Frankfurt am Main

DeMause L (1994) Die Geschichte der Kindheit ist ein Alptraum, aus dem wir gerade erst erwachen. In: Häsing H, Janus L (Hrsg.) Ungewollte Kinder. 236-250. Rowohlt Reinbek 
Diekema D (2010) Support for female circumcision stirs controversy in US. Interview by Simon Mann. Online unter: http://www.smh.com.au/lifestyle/diet-and-fitness/support-for-femalecircumcisionstirscontroversy-in-us-20100521-w1uz.html\#ixzz1tnUUz3jg (abgerufen am 02.10.2013)

Eckert M (2013) Ist das Beschneidungsgesetz verfassungswidrig? Passauer Strafrechtler kritisiert Geringschätzung der Kinderrechte - "Gesetz ebnet Mädchenbeschneidern den Weg“. Passauer Neue Presse 02.02.2013

Egle UT, Hardt I (2005) Pathogene und protektive Entwicklungsfaktoren für die spätere Gesundheit. In: Egle UT, Hoffmann SO, Joraschky P (Hrsg.) Sexueller Missbrauch, Misshandlung, Vernachlässigung: Erkennung, Therapie und Prävention der Folgen früher Stresserfahrungen. 20-43. Schattauer Stuttgart

Enosch I (2012) Ein Akt der Vergewaltigung. Ein Interview von Susanne Knaul. Online unter: http://www.taz. de/!97492 (abgerufen am 02.10.2013)

Franz M (2010) Männliche Genitalbeschneidung und Kindesopfer. Psychohistorische Aspekte eines archaischen Genitaltraumas. In: Borkenhagen A, Brähler E (Hrsg.) Intimmodifikationen. 183-214. Psychosozial-Verlag Gießen

Frisch M, Lindholm M, Grønbæk M (2011) Male circumcision and sexual function in men and women: a surveybased, cross-sectional study in Denmark. International Journal of Epidemiology 40, 1367-1381

Freedman A (2012) Fleshing out change on circumcision. Interview by Ted Merwin. Online unter: http:// www.thejewishweek.com/features/new-york-minute/fleshing-out-change-circumcision (abgerufen am 02.10.2013)

Gray RH, Kigozi G, Serwadda D, Makumbi F, Nalugoda F, Watya S, Moulton L, Chen MZ, Sewankambo NK, Kiwanuka N, Sempijja V, Lutalo T, Kagayii J, Wabwire-Mangen F, Ridzon R, Bacon M, Wawer MJ (2009) The effects of male circumcision on female partners' genital tract symptoms and vaginal infections in a randomized trial in Rakai, Uganda. American Journal of Obstetrics \& Gynecology 200(1), 42.e1-7

Gray RH, Kigozi G, Serwadda D, Makumbi F, Watya S, Nalugoda F, Kiwanuka N, Moulton LH, Chaudhary MA, Chen MZ, Sewankambo NK, Wabwire-Mangen F, Bacon MC, Williams CF, Opendi P, Reynolds SI, Laeyendecker 0, Quinn TC, Wawer M) (2007) Male circumcision for HIV prevention in men in Rakai, Uganda: a randomised trial. Lancet 369(9562), 657-666

Gray RH, Serwadda D, Kong X, Makumbi F, Kigozi G, Gravitt PE, Watya S, Nalugoda F, Ssempijja V, Tobian AA, Kiwanuka N, Moulton LH, Sewankambo NK, Reynolds SI, Quinn TC, Iga B, Laeyendecker O, Oliver AE, Wawer MJ (2010) Male circumcision decreases acquisition and increases clearance of high-risk human papillomavirus in HIV-negative men: a randomized trial in Rakai, Uganda. Journal of Infectious Diseases 201, $1455-1462$

Hallett TB, Baeten IM, Heffron R, Barnabas R, de Bruyn G, Cremin İ, Delany S, Garnett GP, Gray G, Johnson L, McIntyre I, Rees H, Celum C (2011) Optimal uses of antiretrovirals for prevention in HIV-1 serodiscordant heterosexual couples in South Africa: a modelling study. PLoS Med 8(11), e1001123

Herzberg RD (2012) Steht dem biblischen Gebot der Beschneidung ein rechtliches Verbot entgegen? MedR 30, 169-175

Huss W (1994) Die Karthager. 366-386. C.H. Beck München

Kaiser 0 (1970) Einleitung in das Alte Testament. Gerd Mohn Gütersloh

Kelek N (2006) Die verlorenen Söhne. Plädoyer für die Befreiung des türkisch-muslimischen Mannes. 109-122. Kiepenheuer \& Witsch Köln

Muula AS, Prozesky HW, Ronald H, Mataya RH, Ikechebelu II (2007) Prevalence of complications of male circumcision in Anglophone Africa: a systematic review. BMC Urology 7, 4

Okeke LI, Asinobi AA, Ikuerowo OS (2006) Epidemiology of complications of male circumcision in Ibadan, $\mathrm{Ni}$ geria. BMC Urology 6, 21

Pinker S (2011) Gewalt: Eine neue Geschichte der Menschheit. 614-661. Fischer Frankfurt

Püschel K, Cordes 0 (2001) Gewalt gegen Ärzte: Tödliche Bedrohung als Berufsrisiko. Deutsches Ärzteblatt $98(4), A-153 / B-129 / C-125$

Putzke H (2008a) Rechtliche Grenzen der Zirkumzision bei Minderjährigen. Zur Frage der Strafbarkeit des Operateurs nach $₫ 223$ des Strafgesetzbuches. MedR 26, 268-272

Putzke H (2008b) Juristische Positionen zur religiösen Beschneidung. Besprechung von OLG Frankfurt a.M., Beschl. v. 21.8.2007 (4 W 12/07). Neue Juristische Wochenschrift 22, 1568-1570 
Ramos S, Boyle GI (2001) Ritual and medical circumcision among Filipino boys: Evidence of post-traumatic stress disorder. In: Denniston GC, Hodges FM, Milos MF (Hrsg.) Understanding circumcision: A multi-disciplinary approach to a multi-dimensional problem. Kluwer/Plenum New York

Rind M (1998) Menschenopfer: vom Kult der Grausamkeit. Universitätsverlag Regensburg

Sadeh E (2012) Statement von Eran Sadeh, Gründer von Protect the Child, Israel, anläßlich der Pressekonferenz am 12.9.2012. Online unter: http://pro-kinderrechte.de/statement-von-eran-sadeh/ (abgerufen am $02.10 .2013)$

Segal I (2012) Jerome Segal interviewt von Teresa Arrieta: „Als Jude sage ich, Beschneidung ist barbarisch“. Oneline unter: http://www.youtube.com/watch?feature=player_embedded\&v=i8gRNBvCDpU (abgerufen am 02.10.2013)

Schreiber M, Schott GE, Rascher W, Bender AW (2009) Juristische Aspekte der rituellen Zirkumzision. Klinische Pädiatrie 221, 409-414

Serwadda D, Wawer MJ, Makumbi F, Kong X, Kigozi G, Gravitt P, Watya S, Nalugoda F, Ssempiijja V, Tobian AA, Kiwanuka N, Moulton LH, Sewankambo NK, Reynolds SI, Quinn TC, Oliver AE, Iga B, Laeyendecker O, Gray RH (2010) Circumcision of HIV-infected men: effects on high-risk human papillomavirus infections in a randomized trial in Rakai, Uganda. Journal of Infectious Diseases 201(10), 1463-1469

Siegfried N, Muller M, Deeks ||, Volmink I (2009) Male circumcision for prevention of heterosexual acquisition of HIV in men. Cochrane Database of Systematic Reviews 2, CD003362

Stehr M, Putzke H, Dietz H-G (2008) Zirkumzision bei nicht einwilligungsfähigen Jungen: Strafrechtliche Konsequenzen auch bei religiöser Begründung. Deutsches Ärzteblatt 105(34-35), A-1778/B-1535/C-1503

Stehr M, Schuster T, Dietz H-G, Joppich I (2001) Die Zirkumzision - Kritik an der Routine. Klinische Pädiatrie 213(2), 50-55

Taddio A, Katz J, Ilersich L, Koren G (1997) Effect of neonatal circumcision on pain response during subsequent routine vaccination. Lancet 349(9052), 599-603

Tobian AA, Serwadda D, Quinn TC, Kigozi G, Gravitt PE, Laeyendecker O, Charvat B, Ssempijja V, Riedesel M, Oliver AE, Nowak RG, Moulton LH, Chen MZ, Reynolds SI, Wawer MJ, Gray RH (2009) Male circumcision for the prevention of HSV-2 and HPV infections and syphilis. The New England Journal of Medicine 360, 1298-1309

Türcke C (2012) Hyperaktiv!: Kritik der Aufmerksamkeitsdefizitkultur. C.H.Beck München

Viscidi RP, Shah KV (2010) Adult male circumcision: will it reduce disease caused by human papillomavirus? Journal of Infectious Diseases 201, 1447-1449

Weiss HA, Larke N, Halperin D, Schenker I (2010) Complications of circumcision in male neonates, infants and children: a systematic review. BMC Urology 10, 2 